\title{
OPEN Single-cell RNA-seq reveals CD16 monocytes as key regulators of human monocyte transcriptional response to Toxoplasma
}

Anirudh Patir ${ }^{1}$, Anton Gossner ${ }^{2}$, Prakash Ramachandran ${ }^{3}$, Joana Alves ${ }^{2}$, Tom C. Freeman $^{1}$, Neil C. Henderson ${ }^{3}$ Mick Watson $^{1,4}$ \& Musa A. Hassan ${ }^{2,4 \bowtie}$

Monocytes are among the major myeloid cells that respond to Toxoplasma, a ubiquitous foodborne that infects $\geq 1$ billion people worldwide, in human peripheral blood. As such, a molecular understanding of human monocyte-Toxoplasma interactions can expedite the development of novel human toxoplasmosis control strategies. Current molecular studies on monocyte-Toxoplasma interactions are based on average cell or parasite responses across bulk cell populations. Although informative, population-level averages of monocyte responses to Toxoplasma have sometimes produced contradictory results, such as whether CCL2 or IL12 define effective monocyte responses to the parasite. Here, we used single-cell dual RNA sequencing (scDual-Seq) to comprehensively define, for the first time, the monocyte and parasite transcriptional responses that underpin human monocyte-Toxoplasma encounters at the single cell level. We report extreme transcriptional variability between individual monocytes. Furthermore, we report that Toxoplasma-exposed and unexposed monocytes are transcriptionally distinguished by a reactive subset of $C D 14^{+} \mathrm{CD} 16^{-}$monocytes. Functional cytokine assays on sorted monocyte populations show that the infection-distinguishing monocytes secrete high levels of chemokines, such as CCL2 and CXCL5. These findings uncover the Toxoplasma-induced monocyte transcriptional heterogeneity and shed new light on the cell populations that largely define cytokine and chemokine secretion in human monocytes exposed to Toxoplasma.

A majority of lethal human pathogens spend a significant part of their life-cycle inside immune cells, mostly monocytes and macrophages ${ }^{1,2}$. The intracellular lifestyle potentially enable these pathogens to not only evade host immune factors but also antimicrobial therapy ${ }^{2}$. As such, a molecular understanding of pathogen encounters with host immune cells has the potential to identify novel therapeutic targets. Current knowledge on host-pathogen interactions is largely based on experiments performed on bulk populations of host and/or pathogen cells. However, host-pathogen interactions are mostly a single cell problem involving dynamic host and pathogen gene regulatory programs that often produce distinct outcomes in individual cells within a host. For example, bacteria encounters with macrophages from the same individual can simultaneously produce macrophages that; (1) are resistant to infection, (2) undergo programmed cell death to release the bacteria; (3) kill the ingested bacteria, and (4) become infected and allow bacterial growth or persistence ${ }^{3}$. Similar intraindividual heterogenous infection outcomes have been observed in vitro during viral infections $s^{4,5}$ and in vivo during active tuberculosis, during which sterilized and active lesions occur simultaneously in the same host ${ }^{6}$. Phenotypically distinct variants of the same pathogen, such as dormant and actively replicating Mycobacterium, have also been isolated from the same infected host ${ }^{7}$. Although the molecular mechanisms underpinning these distinct intraindividual infection outcomes are largely unknown, it is plausible that they impact disease pathogenesis and antimicrobial therapy ${ }^{8}$. Thus, to understand the biology of infectious diseases and develop effective control strategies, it is important to

\footnotetext{
${ }^{1}$ Division of Genetics and Genomics, The Roslin Institute, University of Edinburgh, Edinburgh EH25 9RG, UK. ${ }^{2}$ Division of Infection and Immunity, The Roslin Institute, University of Edinburgh, Edinburgh EH25 9RG, UK. ${ }^{3}$ Centre for Inflammation Research, The Queen's Medical Research Institute, The University of Edinburgh, Edinburgh EH16 4TJ, UK. ${ }^{4}$ Centre for Tropical Livestock Genetics and Health, The University of Edinburgh, Edinburgh EH25 9RG, UK. ${ }^{凶}$ email: musa.hassan@roslin.ed.ac.uk
} 
consider the overall outcome of an infection as a manifestation of multiple distinct infection outcomes occurring simultaneously in individual cells within a host.

Toxoplasma gondii, the etiological agent for toxoplasmosis, is a zoonotic protozoan that infects virtually all warm-blooded vertebrates ${ }^{9}$. In human peripheral blood, monocytes are among the major myeloid cells that respond to the parasite by secreting interleukin 12 (IL12) ${ }^{10}$, which is required to induce the production of the indispensable anti-Toxoplasma interferon-gamma (IFN $\gamma)$ cytokine ${ }^{11}$. Although usually studied in bulk host and/or parasite cells, when Toxoplasma interacts with monocytes from the same host, several possible outcomes can occur simultaneously to produce distinct monocyte and parasite subpopulations. The parasite can either enter the cell via active invasion to reside within a non-fusogenic parasitophorous vacuole (PV) or be taken up via phagocytosis to reside in a phagosome $e^{10,12}$. In certain cases, phagocytosed parasites can subsequently escape the phagosome to establish a PV ${ }^{13}$. Although not actively infected, some monocytes can also be exposed to, and manipulated by, secreted parasite factors through contact-dependent injection of parasite molecules ${ }^{14}$. Some infected monocytes can undergo programmed cell death ${ }^{15}$ thereby killing the parasite within, while some uninfected bystander monocytes can be activated to produce high levels of IL12 ${ }^{16}$. There are multiple ways to achieve each of these infection outcomes, further expanding the number of molecular pathways that may be regulating the outcome of monocyte-Toxoplasma encounters. Additionally, among canonical human monocyte subsets (classical, $\mathrm{CD} 14^{+} \mathrm{CD} 16^{-}$; intermediate, $\mathrm{CD} 14^{+} \mathrm{CD} 16^{+}$and; non-classical, $\mathrm{CD} 14^{+} \mathrm{CD} 16^{+}$) effective host response to the parasite, defined by the production of IL12, is reportedly restricted to $\mathrm{CD} 16^{+}$monocytes that phagocytose the parasite ${ }^{10}$. Although informative, probably due to averaging of diverse sets of cell responses, cell population-level studies on monocyte-Toxoplasma encounters have sometimes presented contradictory results. For example, a recent study reported that innate recognition of Toxoplasma in human peripheral blood monocytes was characterised mostly by CCL $2^{17}$, rather than the previously reported IL $12^{10}$ or IL $1 \beta^{15,18}$. Importantly, unlike IL12 secretion that required phagocytic parasite uptake by the monocytes ${ }^{10}$, CCL2 secretion was dependent on a soluble effector ${ }^{17}$. To resolve such discrepancies and characterize heterogeneous monocyte-Toxoplasma interactions, we need to go beyond population averages and define single cell responses that when combined represent the entire monocyte and parasite responses.

Single cell RNA sequencing (scRNA-seq), which captures transcript abundance in single cells ${ }^{19}$, can provide the high-resolution needed to resolve transcriptional programmes underlying disparate host-pathogen interactions. scRNA-seq has so far been used to characterise, among others, the transcriptional profiles underpinning: intra-individual disparate macrophage response to Salmonella ${ }^{3}$; latent and reactivating HIV-infected human $\mathrm{CD} 4+\mathrm{T}$ cells ${ }^{20}$ from the same donor; and the sexual commitment and development of individual Plasmodium parasites ${ }^{21,22}$. While host-pathogen interactions involve two organisms with distinct transcriptomes, scRNA-seq profiling of host-pathogen interaction is typically restricted to a single organism at a time. Single-cell dual RNAsequencing (scDual-Seq), a hybrid of scRNA-Seq and dual RNA-seq ${ }^{23}$, can be used to simultaneously monitor the host and pathogen transcriptomes during an infection ${ }^{24,25}$.

Here, we exploit the fact that both Toxoplasma and human mRNAs are polyadenylated and can be simultaneously profiled using standard scRNA-seq protocols to investigate the transcriptional hallmarks of Toxoplasma interactions with monocytes from the same donor. We report significant heterogeneity among individual monocytes and parasites. Furthermore, we find that Toxoplasma-exposed and control monocytes are transcriptionally distinguished by non-classical monocytes and a novel subset of reactive classical monocytes.

\section{Results}

Defining the single-cell transcriptome of Toxoplasma-exposed human monocytes. In this study, we used single-cell RNA sequencing (scRNA-seq; 10X Genomics) to perform unbiased transcriptional analysis of human monocytes exposed to Toxoplasma for $1 \mathrm{~h}$. We chose to a $1 \mathrm{hr}$ infection because of a previous report that showed Toxoplasma-induced expression of key monocyte genes, including IL1 $\beta$, occur as early as $1 \mathrm{hr}$ post infection, followed by a gradual decrease in expression ${ }^{15}$. We conducted several pre-processing steps (Materials and Methods) including quality control, normalization, and scaling ${ }^{26}$ to remove potential technical bias. The processed control (unexposed) monocyte expression matrix contained 3136 cells and 12023 genes while the Toxoplasma-exposed monocyte data contained 1352 cells and 10795 genes. Unsupervised graph-based clustering partitioned the unexposed monocytes into two clusters: hU1 (human-unexposed) and hU2, which correspond to the non-classical $\left(\mathrm{CD} 14^{+} \mathrm{CD} 16^{+}\right)$and classical $\left(\mathrm{CD} 14^{+} \mathrm{CD} 16^{-}\right)$monocyte subsets, respectively ${ }^{27}$, (Fig. 1A). The Toxoplasma-exposed monocytes clustered into three distinct groups: hE1 (human-exposed), which is composed entirely of non-classical monocytes; hE2; and hE3, both of which were made mostly up of classical monocytes (Fig. 1B). The proportions of classical and non-classical cells identified in both conditions (Fig. 1A,B) is largely consistent with reports on the composition of circulating human monocytes ${ }^{28}$.

Next we used a Gini coefficient metric, a measure of population inequality ranging from zero (complete equality) to one (complete inequality) to evaluate gene expression variability within the monocyte subsets. Cells from the hE3 cluster showed significantly $(F D R<0.05$, medium Gini index $=0.93)$ higher Gini index relative to the other clusters (Fig. 1C). In scRNA-seq mRNAs from the parasite and the monocyte it infects are tagged with the same cell barcode, which enables the reliable identification of infected monocytes (contain parasite mRNA) and uninfected (do not contain parasite RNA). Thus, to determine whether the differences between monocyte clusters was due to differences in the number of infected monocytes, we examined the proportion of infected cells in each of the Toxoplasma-exposed monocyte clusters. There were no significant differences in the proportion of infected cells in each cluster (Fig. 1D). However, it is worth noting that by injecting its effector proteins into host cells, Toxoplasma can modulate the transcriptome of host cells without truly infecting them. As such, part of what we consider exposed-uninfected (bystander) cells in this study may include cells that are injected with parasite effector proteins or infected cells from which we were unable to recover parasite mRNA (for a variety 
A
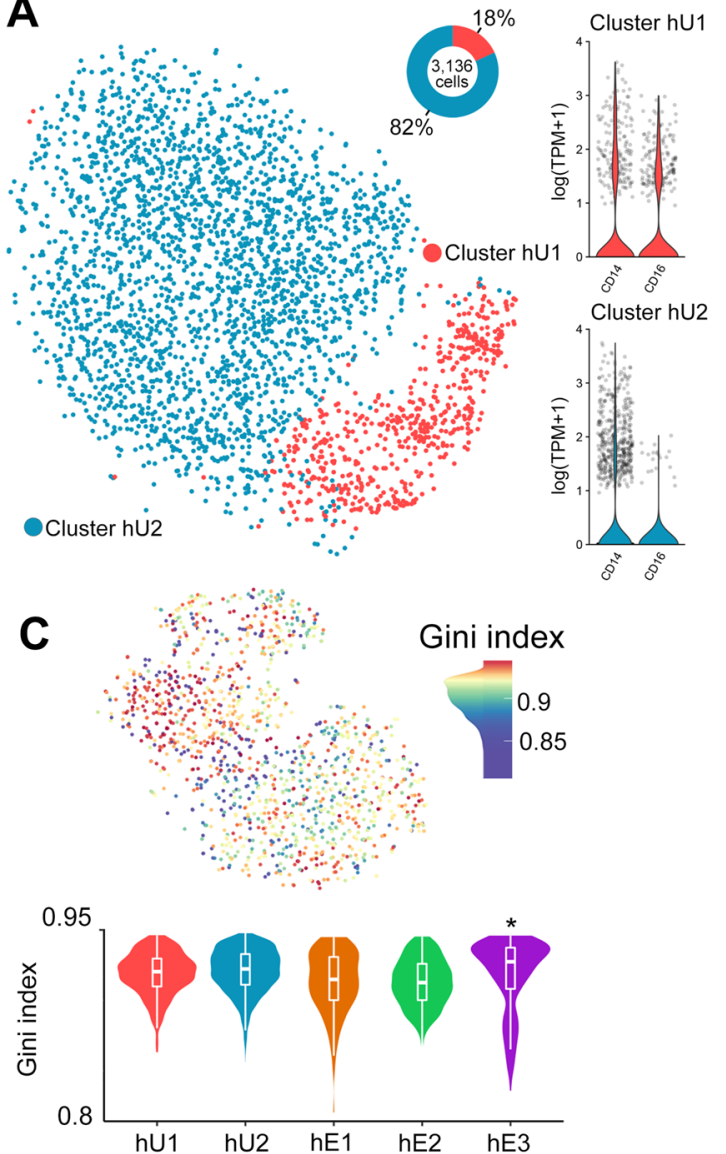

B
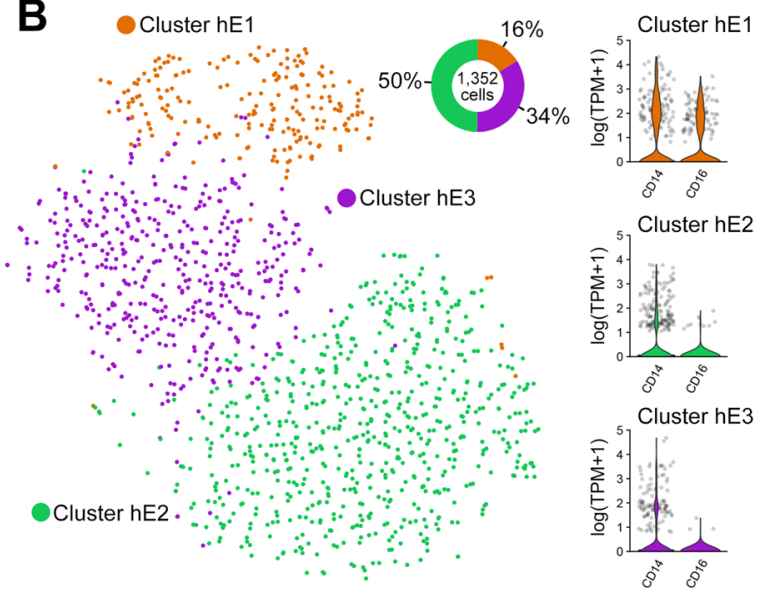

D

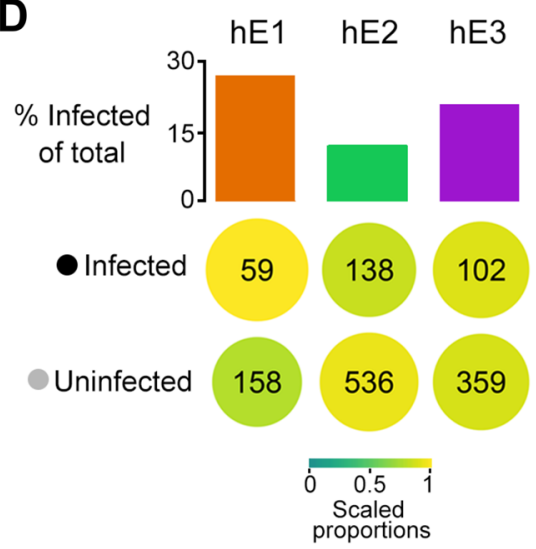

Figure 1. Transcriptional heterogeneity within Toxoplasma-exposed and unexposed human monocytes: $t$ SNE plots of monocyte clusters of (A) unexposed (hU1 \& hU2) and (B) Toxoplasma-exposed (hE1, hE2, and hE3) to human monocytes, including non-classical and classical monocytes. For each condition the proportion of cells within a cluster and the expression of monocyte subset marker genes (CD14 and CD16) are shown. (C) A tSNE plot (Top panel) of Toxoplasma-exposed monocytes showing the Gini coefficients for each cell. Higher Gini index represent higher variation of expression across genes. Violin plots of the Gini coefficients for each monocyte cluster from Toxoplasma-exposed and unexposed monocytes (Bottom panel). (D) The proportion of infected and uninfected cells within each cluster. Additionally, highlighting preferential infectivity of clusters (colour and size) while scaling for the total infected/uninfected cells and the number of cells within a cluster. $\mathrm{TPM}=$ Transcript per Million reads; ${ }^{*} P$ value $\leq 0.05$.

potential of technical issues, such as sequencing depth). Nevertheless, when we restricted the unsupervised hierarchical clustering to cells that contain, or do not contain parasite mRNA, we did not observe new clustering patterns (not shown). Taken together, we find that individual monocytes from the same donor respond variably to Toxoplasma at the transcriptional level and that this transcriptional heterogeneity is more pronounced in the classical monocyte $\left(\mathrm{CD} 14^{+} \mathrm{CD} 16^{-}\right)$subset.

A subset of CD16 monocytes transcriptionally distinguish Toxoplasma-exposed and unexposed human monocytes. Previous immunological and parasitological assays in human monocytes have reported differential response to Toxoplasma between classical and non-classical monocyte subsets, as well as between monocytes that are actively invaded and those that take up the parasites through phagocytosis ${ }^{10,29}$. Having observed transcriptional heterogeneity in the Toxoplasma-exposed monocytes, we determined whether a distinction between responsive and unresponsive monocyte subsets can be discerned at the transcriptional level. A $t$-distributed stochastic neighbour embedding ( $t \mathrm{SNE}$ ) plot, which depicts the similarity between cells based on their gene expression, of the combined Toxoplasma-exposed and unexposed monocytes scRNA-seq data showed that the exposed and control cells are clearly transcriptionally distinguished by the hE3 cluster (Fig. 2). As indicated above, although hE3 distinguished Toxoplasma-exposed and unexposed monocytes, there was no evidence that cells in this cluster contained more parasite genes than the hE1 or hE2 clusters.

To gain further insights into the Toxoplasma-exposed monocytes, we sub-divided cells in each cluster into infected (contain parasite mRNA) and uninfected (do not contain parasite mRNA) and performed differential expression (DE) analysis between the clusters. 711 non-redundant genes were differentially expressed (S1A), of which 314 were highly expressed in specific monocyte clusters (Fig. 3A). 218 genes were highly expressed in the hE3 cluster, of which 22 were highly expressed in the infected and 196 in the uninfected monocytes (Fig. 3A and 

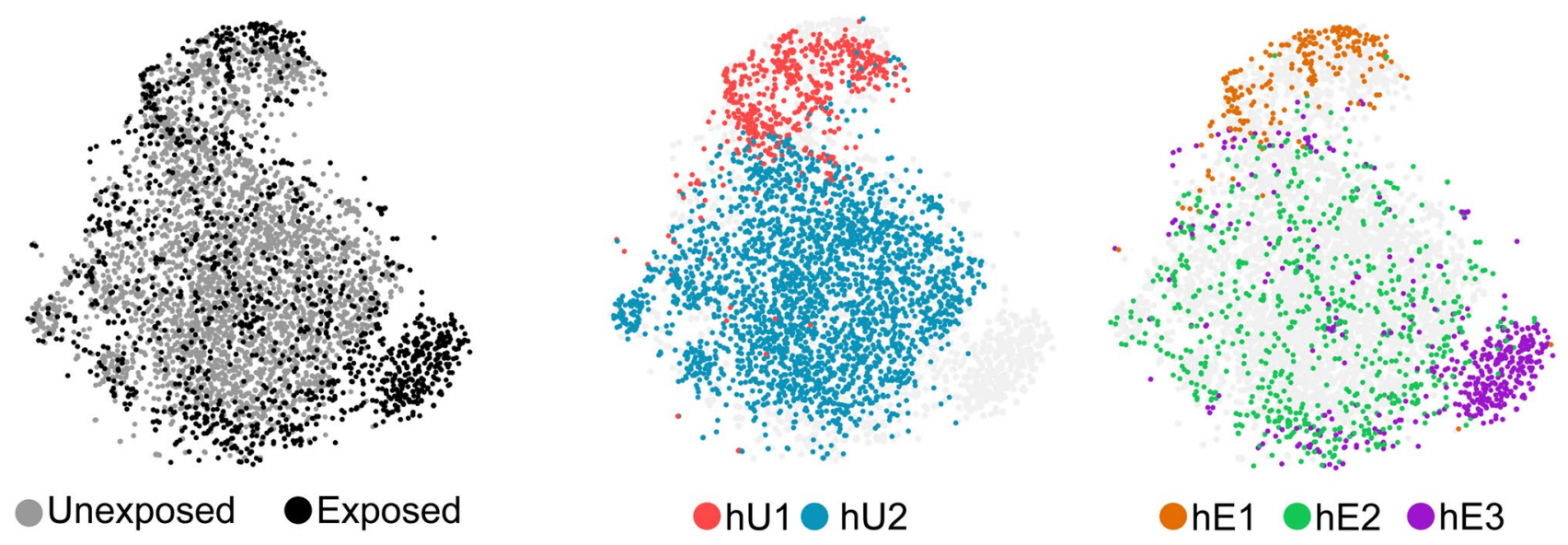

Figure 2. A unique subset of CD16- monocytes distinguish exposure to Toxoplasma: $t$ SNE plots of the combined Toxoplasma-exposed and unexposed monocytes datasets overlaid with the monocyte clusters identified from each condition (Centre and left panels).

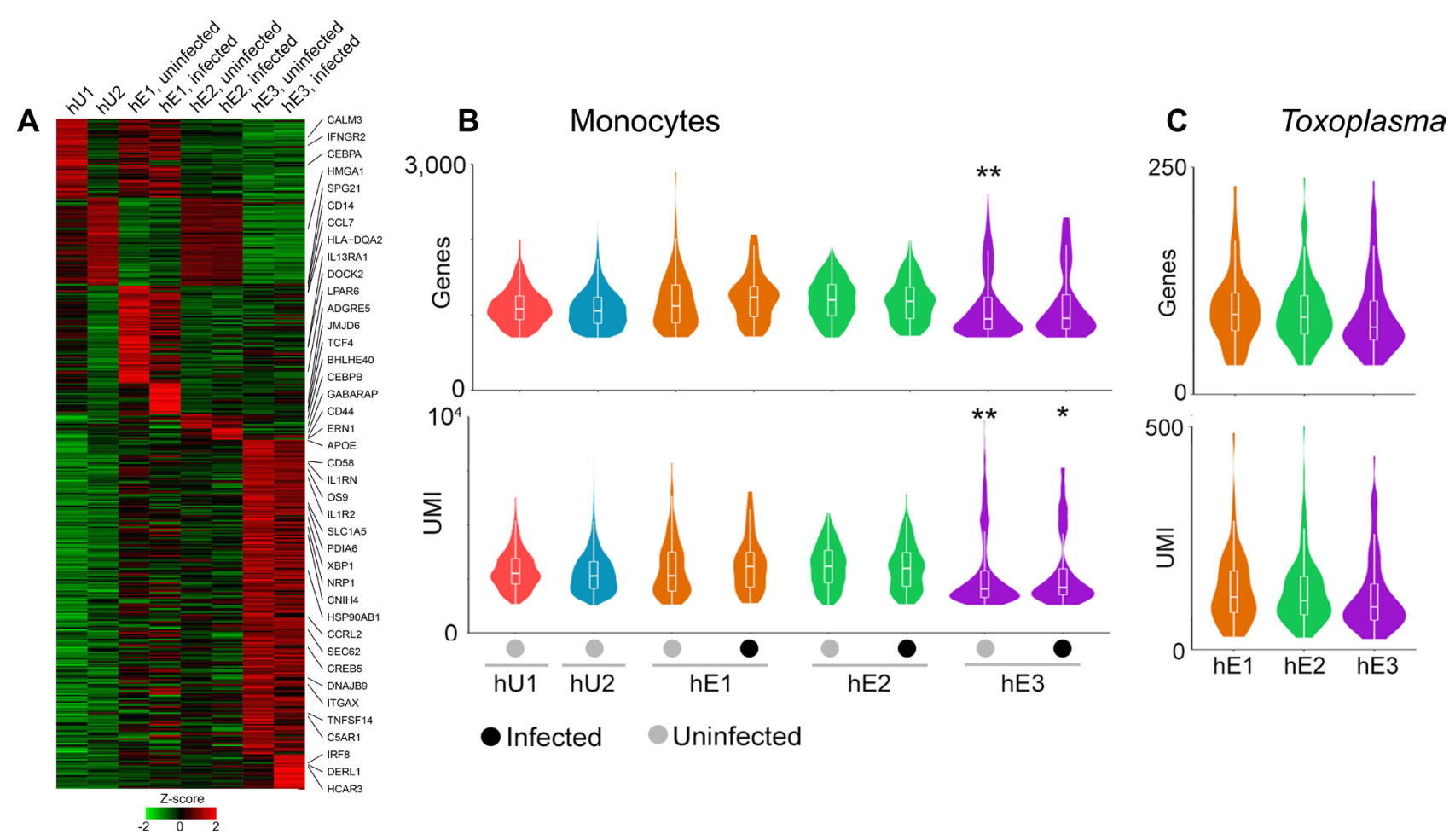

Figure 3. Gene expression across monocyte subsets: (A) A heatmap showing the average expression of the 418 DE genes for each monocyte cluster in Toxoplasma-exposed and unexposed monocytes. The number of genes and reads mapped to $(\mathbf{B})$ Monocytes and $(\mathbf{C})$ invading Toxoplasma. ${ }^{\star}$ Significance at $F D R<0.05,{ }^{*}$ significance at $F D R<0.01$.

S1A), suggesting that the transcriptional heterogeneity in the hE3 cluster is driven by the uninfected bystander cells. Interestingly, the number of human genes and reads were significantly lower (FDR $<0.05)$ in hE3 infected and uninfected cells relative to other groups (Fig. 3B). However, there was no significant difference in the number of parasite genes within the monocyte clusters (Fig. 3C). Similarly, out of the 80 highly expressed genes in the hE1 cluster, 61 (76\%) were expressed in both the infected and uninfected cells. Despite being like the hE3 cluster in monocyte subset composition, the hE2 cluster had the least number (16) of highly expressed DE genes.

To functionally annotate DE genes in hE1 or hE3 clusters, enrichment analysis was performed. The top Gene Ontology (GO) terms enriched in DE genes that were highly expressed in both the uninfected and infected cells in hE3 cluster included innate immune associated terms, such as "immune effector process" $\left(F D R=3.48 \times 10^{-4}\right)$, "myeloid cell activation involved in immune response" $\left(F D R=4.10 \times 10^{-7}\right)$, and "cell activation involved in immune response" $\left(F D R=2.95 \times 10^{-6}\right)($ Table $1 \& \mathrm{~S} 1 \mathrm{~B})$. Among the DE genes in the hE3 cluster 


\begin{tabular}{|l|l|l|l|}
\hline & & \multicolumn{2}{l|}{ Entities } \\
\cline { 3 - 4 } Cluster & Biological process & q-value & Found \\
\hline hE1 infected & Negative regulation of dendritic cell differentiation & $1.34 \mathrm{E}-02$ & $2 / 8$ \\
\hline hE1 infected & Negative regulation of T cell mediated cytotoxicity & $1.88 \mathrm{E}-02$ & $2 / 7$ \\
\hline hE1 infected & Regulation of dendritic cell differentiation & $9.34 \mathrm{E}-02$ & $2 / 15$ \\
\hline hE1 infected & Immune effector process & $1.12 \mathrm{E}-01$ & $7 / 1338$ \\
\hline hE1 uninfected & Regulation of immune system process & $2.51 \mathrm{E}-07$ & $24 / 1787$ \\
\hline hE1 uninfected & Immune effector process & $3.25 \mathrm{E}-07$ & $21 / 1338$ \\
\hline hE1 uninfected & Cell activation involved in immune response & $8.04 \mathrm{E}-06$ & $15 / 739$ \\
\hline hE1 uninfected & Myeloid cell activation involved in immune response & $1.92 \mathrm{E}-04$ & $12 / 557$ \\
\hline hE3 infected & Rough endoplasmic reticulum membrane & $3.78 \mathrm{E}-02$ & $2 / 29$ \\
\hline hE3 infected & Cellular response to molecule of bacterial origin & $5.58 \mathrm{E}-02$ & $4 / 233$ \\
\hline hE3 infected & Protein processing in endoplasmic reticulum & $7.72 \mathrm{E}-02$ & $3 / 166$ \\
\hline hE3 infected & Regulation of protein modification by small protein conjugation or removal & $7.92 \mathrm{E}-02$ & $4 / 255$ \\
\hline hE3 uninfected & Neutrophil activation involved in immune response & $1.91 \mathrm{E}-08$ & $25 / 491$ \\
\hline hE3 uninfected & Myeloid cell activation involved in immune response & $4.84 \mathrm{E}-08$ & $26 / 557$ \\
\hline hE3 uninfected & Cell activation involved in immune response & $9.44 \mathrm{E}-07$ & $28 / 739$ \\
\hline hE3 uninfected & Response to endoplasmic reticulum stress & $1.19 \mathrm{E}-06$ & $18 / 294$ \\
\hline
\end{tabular}

Table 1. The top 4 most enriched biological processes in each cluster.

were pro-inflammatory chemokines that are known to be induced in human peripheral blood mononuclear cells and monocytes exposed to Toxoplasma ${ }^{17}$, such as CXCL8, (for infected, fold change $=1.5, F D R=2.11 \times 10^{-3}$ ; uninfected, fold change $=2.9, F D R=7.61 \times 10^{-59}$ ). GO terms enriched in the uninfected cells in the hE3 cluster included "response to endoplasmic reticulum unfolded protein" $\left(F D R=9.91 \times 10^{-8}\right)$ and "endoplasmic reticulum part" $\left(F D R=6.45 \times 10^{-15}\right)$. Consistent with the GO enrichment analysis, the $\mathrm{x}$-box binding protein $(X B P 1)$, a transcription factor that is critical for the induction of genes required for resolving endoplasmic reticulum stress induced by the accumulation of unfolded proteins ${ }^{30}$, was one of the highly expressed DE genes in the uninfected cells in hE3 cluster (Fig. 3A). Besides XBP1, DE genes highly expressed in the uninfected cells in hE3 included other unfolded protein response (UPR)-related genes such as DNAJB9 (a target of XBP1), OS9, and PDIA6 (Fig. 3A). GO terms enriched in the infected cells in the hE3 cluster included "cellular response to LPS" $(F D R=5.05 \mathrm{E}-2)$ and "cellular response to molecules of bacterial origin" $(F D R=5.58 \mathrm{E}-2)$. Enrichment analysis for the hE1 cluster also revealed immune response associated terms such as, "regulation of immune system process" $\left(\mathrm{FDR}=7.53 \times 10^{-4}\right)$ and "defence response" $\left(\mathrm{FDR}=7.71 \times 10^{-3}\right)$. The top cellular component terms enriched in the uninfected cells in the hE1 cluster included "lysosome" $\left(F D R=1.5 \times 10^{-4}\right)$ and "lytic vacuole" $\left(F D R=1.5 \times 10^{-4}\right)$. Congruent to observations in the THP-1 cell line (a human monocyte cell line that replicates most Toxoplasma infection phenotypes observed in primary human monocytes) that are infected or separated from Toxoplasma in transwell ${ }^{17}$, CCL2 was DE in both the infected $\left(1.9\right.$-fold, $\left.F D R=7.57 \times 10^{-3}\right)$ and uninfected (3.3-fold, $\left.F D R=3.35 \times 10^{-17}\right)$ cells in the hE1 cluster (S1A). The expression of CCL2 in Toxoplasma-exposed monocytes is reportedly initiated by the S100 calcium-binding protein A11 (S100A11) ${ }^{17}$. However, unlike the other members of the S100 family, including S100A4, 6 and 9 that were DE in hE1, S100A11 was not. Considering all DE genes, those highly expressed in hE1 included members of the MHC class 2 family and genes associated with interferon response (IFIT, IRF and OAS gene families). Though few genes were DE solely in hE2, the cluster showed a high expression of immune related genes that were also expressed in other groups, including CCL3, CCL4, NFKBIA and IL1 $\beta$. Certain DE genes expressed in hE3 and hE1 such as members of the MHC class 1 , cathepsins and proteasome complex, were not expressed in the hE2 cluster. In summary we reveal an unprecedented level of transcriptional heterogeneity in Toxoplasma-exposed monocytes and that transcriptional response to the parasite at $1 \mathrm{~h}$ post exposure is defined mostly by a subset CD16- monocytes.

Monocyte response to Toxoplasma is defined by chemokine secretion. The observation that a subset of $\mathrm{CD}_{16} 6^{-}$monocyte subset (hE3) transcriptionally distinguish Toxoplasma-exposed and unexposed monocytes prompted us to investigate whether the transcriptional heterogeneity translate into a distinctive infection phenotype. To explore this, we used monoclonal antibodies against the cell surface protein product of the SLC3A2 (CD98) gene that is highly expressed in the hE3 cluster (Fig. 4A) to sort Toxoplasma-exposed monocytes followed by functional assays. First, we explored the specificity of CD98 as marker for hE3 by using qPCR to quantify the expression of CD98 in Toxoplasma-exposed $\mathrm{CD}_{16}{ }^{+}$and $\mathrm{CD} 16^{-}$relative to the corresponding controls. Consistent with observations from the scRNA-seq, the expression of CD98 was significantly $(P \leq 0.01)$ high in $\mathrm{CD}^{-} 6^{-}$relative to $\mathrm{CD}_{16}{ }^{+}$(Fig. $4 \mathrm{~B}$ ). Next, we used a human multiplex cytokine array to measure the level of several cytokines and chemokines, including CCL2 and IL12, in cell-free supernatants from the sorted CD $98^{+}$monocytes. CD98- monocytes were used as controls. The sorted CD $98^{+}$cells secreted significantly more CXCL5, CCL2 and MIP1, but less IL12 and IL6, compared to CD98- monocytes (Fig. 4C). Previously, CCL2 was reported to be highly expressed in human monocytes exposed to Toxoplasma ${ }^{17}$. Thus, we determined the correlation between the expression of CD98 and CCL2 in different human monocyte subsets. Using qPCR to quantify 

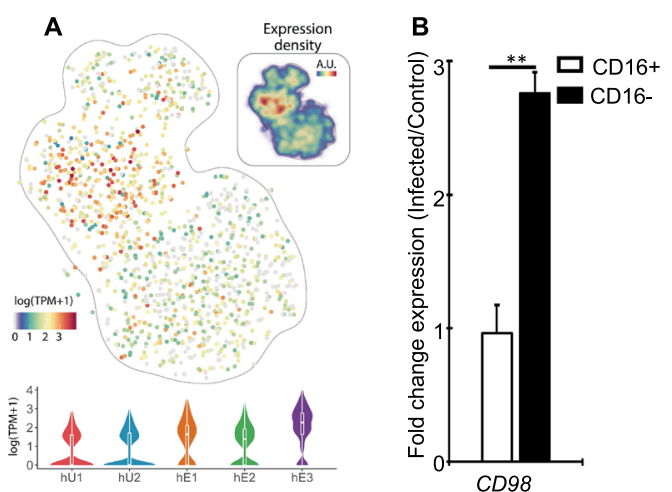

C
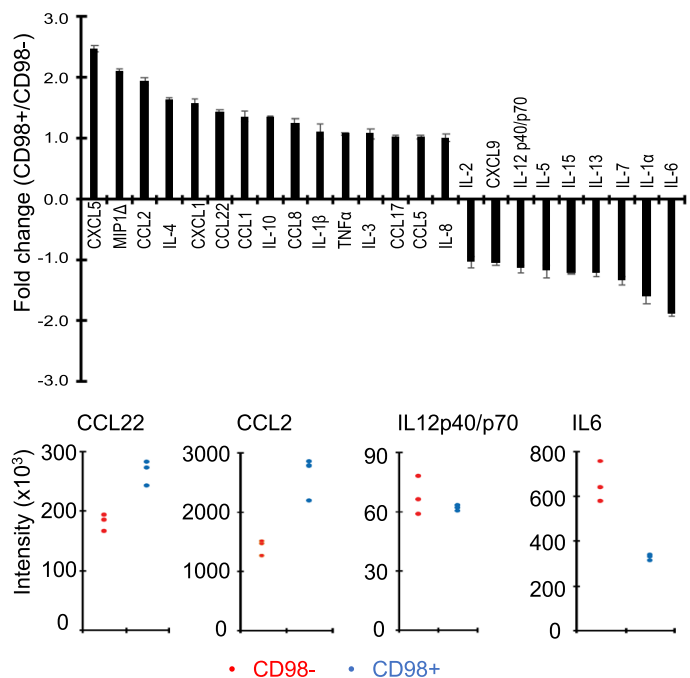

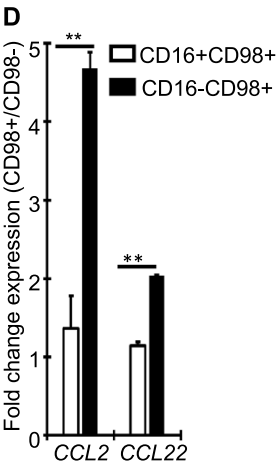

Figure 4. A functional analysis of infection-distinguishing Toxoplasma-exposed monocytes: (A) A heatmap showing the expression of CD98 in the Toxoplasma-exposed (hE) and naive (hU) monocytes clusters based on the scRNA-seq data. Inset is the expression density in arbitrary units (A.U). (B) The expression of CD98 in Toxoplasma-exposed CD16 + and CD16- monocytes, relative to naïve controls. (C) Fold changes of cytokine and chemokine levels in sorted $\mathrm{CD} 8^{+}$, relative to control (CD98 $8^{-}$monocytes averaged across (upper panel) or plotted for individual (lower panel) donors. Each dot in the lower panel represents an individual donor. (D). The expression of CCL2 and CCL22 in sorted $\mathrm{CD} 98^{+} \mathrm{CD} 16^{+}$or $\mathrm{CD} 16^{-}$, relative to the corresponding CD98 ${ }^{-}$ monocytes. Values are mean \pm s.e of three independent biological repeats. ${ }^{\star \star} P \leq 0.01$.

the expression of CCL2 and CCL22 in Toxoplasma-exposed CD16 $/ \mathrm{CD} 98^{+}$and $\mathrm{CD} 16^{-} / \mathrm{CD} 98^{+}$monocytes, we observed enhanced expression of both chemokines in CD98 $8^{+}$monocytes (Fig. 4D). Interestingly, we could not CD98 ${ }^{+}$cells in naïve monocytes in cytometry. The level of IL1 $\beta$, known to be secreted by monocytes in response to Toxoplasma ${ }^{15}$, was induced in $\mathrm{CD} 8^{+}$. Therefore, Toxoplasma-induced transcriptional heterogeneity observed in human monocytes can be linked to specific Toxoplasma-induced monocyte phenotypes at the protein level.

Transcriptional heterogeneity ofToxoplasma exposed to monocytes. Host-pathogen encounters are highly dynamic processes modulated by both host- and pathogen-derived factors. Therefore, we investigated whether parasites display different transcriptional profiles in individual monocytes. After data pre-processing and quality control we recovered 873 parasites, of which 299 shared cell barcodes with individual monocytes. In total, the 299 Toxoplasma cells expressed 2556 genes. We initially grouped parasites based on the type of monocyte they infected; however, no DE genes were identified for any of these groups. Subsequently, parasites were clustered into three distinct groups (hT1, hT2 and, hT3) based on their gene expression (Fig. 5A). Varying abundance of parasites were observed between clusters, the largest being hT1 (585 parasites) and the smallest hT3 (109 parasites) (Fig. 5B,C). However, in all cases approximately the same proportion, 33-37\% of parasites were linked to monocytes. To determine whether there were differences in transcriptional activity between parasites that were linked to individual monocytes and parasites not linked to individual monocytes for a given cluster, we examined the number of reads and their cognate genes in each cluster. We observed significant differences in the number of RNA-reads and number of detected genes between parasites linked to monocytes and parasite not linked to monocytes in the hT1 and hT2, but not hT3 (Fig. 5D). To identify the genes that underpin the parasite transcriptional heterogeneity in individual monocytes, we performed DE analysis between the parasite clusters. Of the $169 \mathrm{DE}$ genes, 40, 73, and 80 were highly expressed in hT1, hT2 and hT3, respectively (S2A). The top DE genes included several ribosomal proteins, the microneme protein 10 (MIC10), a putative elongation factor 1-alpha (EF-1-ALPHA), and a dense granule protein (GRA12) in hT1; SAG-related sequence (SRS20A), several rhoptry and rhoptry neck proteins such as ROP17 and RON8 in hT2 and; a microtubule-associated protein (SPM1), GAP45, and several hypothetical proteins in hT3. Next, we analysed the biological processes enriched in the DE genes using functional gene enrichment analysis based on the gene ontology terms available in ToxoDB ${ }^{31}$. The hT1 cluster, in which several ribosomal genes were DE, was enriched in "translation" $\left(F D R=1.6 \times 10^{-7}\right)$ and "peptide biosynthetic processes" $\left(F D R=1.6 \times 10^{-7}\right)(\mathrm{S} 2 \mathrm{~B})$. The hT2 and hT3 clusters were enriched in "Apical part of cell" and "Pellicle" terms associated cellular localization $(F D R<0.001)$. In summary, although the parasites exhibit transcriptional heterogeneity, it is not apparent from the current data, that this heterogeneity is driven by the cognate monocyte transcriptional landscape. 

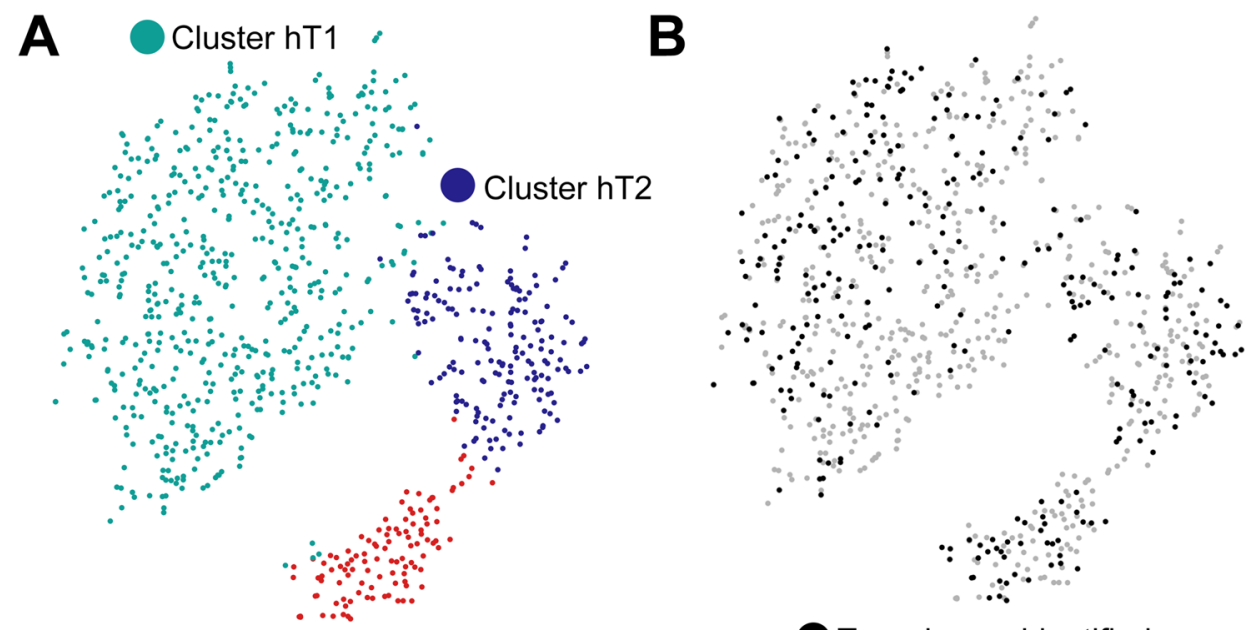

Cluster hT3

C
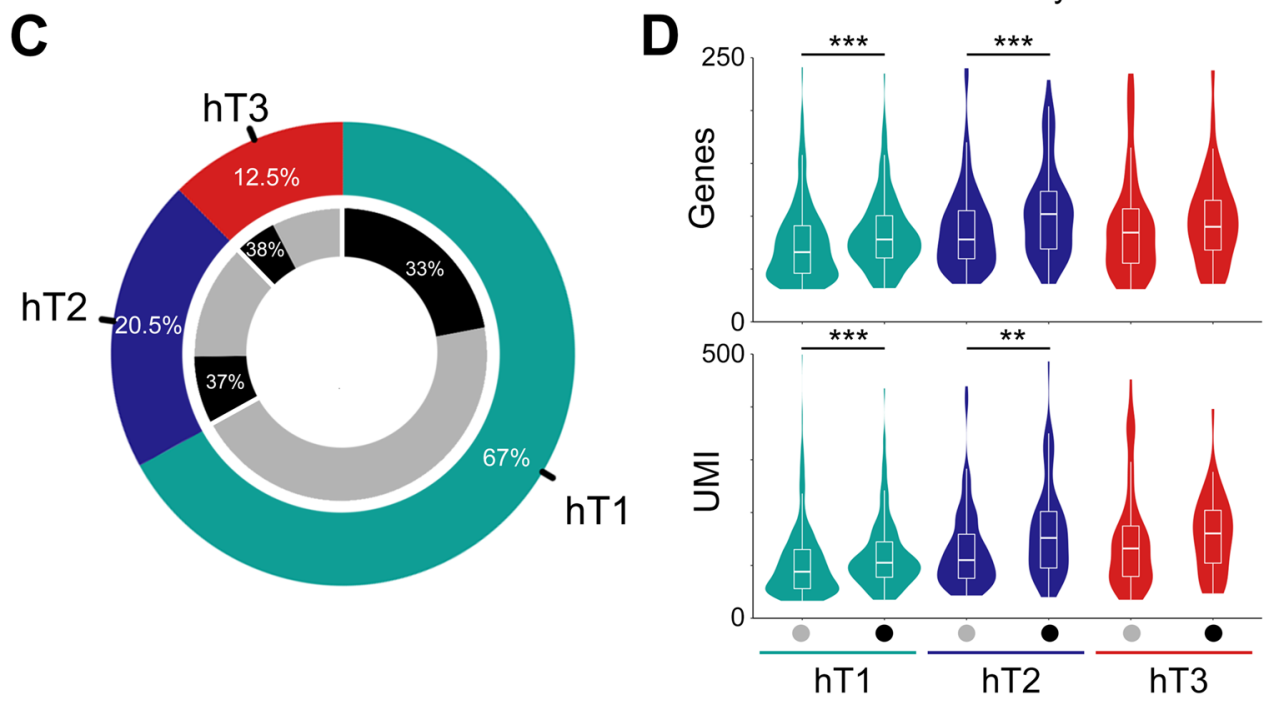

Figure 5. Transcriptional heterogeneity of Toxoplasma exposed to monocytes: (A-C) tSNE and clustering of Toxoplasma parasites with the proportion of cells shown for each cluster (hT1, hT2 and hT3). The inner ring shows the proportion of parasites in each cluster that can be linked accurately to an individual monocyte based on sharing of cell barcodes. (D) The distribution of Toxoplasma RNA-seq reads and Toxoplasma genes expressed based on their clustering and whether they linked to a monocyte (black dot). ${ }^{* *}$ Significance at $F D R<0.01$, $* * *$ significance at $F D R<0.001$.

\section{Discussion}

Herein, we applied a combination of scRNA-seq and phenotyping of sorted cell populations to provide a high dimensional insight into human monocyte-Toxoplasma interactions. We have shown that individual monocytes from the same donor exhibit great transcriptional heterogeneity in response to the parasite and that Toxoplasmaexposed and unexposed cells can be distinguished transcriptionally by a subset of CD16- monocytes, composed of both infected and potential bystander cells. In addition, our results show that the transcriptional heterogeneity translates into functional differences between individual monocyte clusters, including differences in CCL2 and IL1 $\beta$ secretion, which previous studies have reported to be induced in human monocytes exposed to either Toxoplasma or cell-free supernatant from Toxoplasma-infected cells ${ }^{15,17}$.

Previously, CD16 + monocytes were reported to distinguish responsive monocytes, based on IL12 secretion ${ }^{10}$. Here, although CD16 + cells can transcriptionally distinguish Toxoplasma-exposed and unexposed monocytes, as shown in Fig. 2, a clear distinction is only achieved with a subset of CD16- monocytes that express CD98. This discrepancy may be due to several factors. Besides averaging responses in cell populations, which can mask cell-to-cell differences in IL12 secretion, this and the previous study are based on different phenotypes; transcript and protein abundance, which often do not match ${ }^{32}$. Importantly, we investigated monocytes exposed to Toxoplasma for $1 \mathrm{~h}$, rather than $24 \mathrm{~h}$ as in the previous study ${ }^{10}$. Thus, it is plausible that early $(1 \mathrm{~h})$ responses to the parasite are defined mostly by CD16 monocytes while long-term $(24 \mathrm{~h})$ responses are modulated by CD16 ${ }^{+}$ monocytes. Additionally, unlike in the previous study ${ }^{10}$, we seeded the monocytes overnight prior to infection, 
which can reportedly affect the expression of some inflammatory genes including IL $1 \beta^{33}$. As such discrepancy between this and the previous study ${ }^{10}$ could be temporal, rather than functional. Nevertheless, we observed more IL12 in cell-free supernatants from control, compared to sorted hE3 cells, suggesting that significant differences in IL12 secretion are discernible at $1 \mathrm{~h}$ post-infection. Noteworthy, primary human monocyte response to Toxoplasma was recently reported to be defined more by the secretion of chemokines, including CCL2, than IL12 ${ }^{17}$. Consistent with this observation, we observed differential expression of several chemokines between the different Toxoplasma-exposed monocyte clusters. Furthermore, sorted CD98+ monocyte populations that distinguish Toxoplasma-exposed and unexposed monocytes secreted more chemokines, including CCL2 than the corresponding controls. We propose a future temporal scRNA-seq analysis of monocyte-Toxoplasma encounters to determine whether additional monocyte clusters that distinguish Toxoplasma-exposed and unexposed monocyte clusters emerge over time.

Toxoplasma transcriptional heterogeneity is consistent with observations in other intracellular pathogens infecting cells of the monocyte/macrophage lineage, including Salmonella ${ }^{25}$. Toxoplasma can infect phagocytic cells either via active parasite invasion or phagocytic parasite uptake, with the parasite ending up initially in the $\mathrm{PV}$ or phagosome $\mathrm{1}^{10,12,13}$. Monocyte responses to the parasite, including IL12 secretion, is reportedly influenced by the route of parasite entry; only phagocytosed parasites induce IL12 secretion ${ }^{10}$. Although we observed transcriptional segregation within parasite-containing monocyte population at $1 \mathrm{hr}$ post-infection, we did not observe an overrepresentation of parasite-containing cells in individual monocyte clusters (Figure S1). This suggests that, at this early time point, active invasion or phagocytic parasite uptake probably does not define the monocyte transcriptional segregation or that Toxoplasma transcriptional heterogeneity is not modulated by intracellular niche (PV or phagosome). Currently, we lack insight into what role the route of parasite entry play in the monocyte transcriptional segregation, mostly due to a lack of well-defined transcriptional markers of actively invaded and monocytes that phagocytose the parasite. However, we are in the process of generating transcriptional profiles of sorted bulk populations of actively invaded, phagocytic, and truly bystander monocyte subpopulations, which will greatly improve our current analysis. Most genes that were differentially expressed in Toxoplasma-exposed relative to unexposed monocytes were from bystander cells. This may suggest that the responses from bystander cells drive the phenotypic outcome of infection rather than the transcriptional responses in the parasites and monocytes they infect. However, as indicated above, since we identify infected cells by the presence of parasite mRNA in it, it is possible that some of the exposed-uninfected (considered bystander) monocytes are indeed infected, but we were not able to detect parasite mRNA due to technical limitations or are truly uninfected but are injected with Toxoplasma effector proteins. A more in-depth analysis will be possible once we transcriptionally define different subpopulations of Toxoplasma-exposed monocytes, including actively invaded, phagocytic, and exposed-uninfected subpopulations.

\section{Material and methods}

Blood donors and monocyte isolation. Human whole blood was collected from three Toxoplasma-free (confirmed by standard anti-Toxoplasma antibody test in blood [REF]) healthy adult donors who provided written informed consent. Blood was collected with ethical approval from the South East Scotland LREC (ref: 11/ $\mathrm{AL} / 0168)$. All the experimental protocols were approved and conducted in accordance with the health and safety guidelines of, The Roslin Institute's Health and Safety Committee. Monocytes were isolated from peripheral blood mononuclear cells as previously described ${ }^{10}$, to yield $>98 \%$ pure monocytes.

Parasites and infection. RH strain tachyzoites, maintained by serial passage on human foreskin fibroblasts (HFFs; originally obtained from the Boothroyd lab, Stanford University), were used in all infections. Parasites were grown in RPMI (Life Technologies) supplemented with $1 \%$ foetal bovine serum (FBS; Omega Scientific), $2 \mathrm{mM}$ glutamine (Sigma), $10 \mathrm{mM}$ HEPES (pH 7.5; Sigma), and $20 \mu \mathrm{g} / \mathrm{ml}$ gentamicin at $37^{\circ} \mathrm{C}$ in $5 \% \mathrm{CO}_{2}$. The parasites used for monocyte infection were prepared by scraping T-25 flasks containing heavily vacuolated HFFs followed by sequential passage through $25 \mathrm{G}$ and $27 \mathrm{G}$ needles. The released parasites were pelleted by centrifugation at $572 \times \mathrm{g}$ for $7 \mathrm{~min}$, washed in phosphate-buffered saline (PBS; Life Technologies), filtered using a $5 \mu \mathrm{m}$ membrane to exclude host cell debris, and counted. $2 \times 10^{6}$ monocytes, obtained from peripheral blood as previously described ${ }^{10}$, were plated in 6 -well tissue culture plates overnight prior to infection. The cells were left unexposed or exposed to freshly prepared parasites at a multiplicity of infection (MOI) of 1:1, briefly centrifuged to bring the monocytes and parasite into contact and incubated at $37^{\circ} \mathrm{C}$ in $5 \% \mathrm{CO}_{2}$ for $1 \mathrm{~h}$ before processing the cells for single-cell sequencing or flow cytometry.

Single cell RNA-sequencing. Toxoplasma-exposed and unexposed monocytes from one donor were placed on ice, scraped and washed three times in cold PBS supplemented with $0.1 \%$ bovine serum albumin (BSA, Thermo Fisher). The scRNA-seq libraries were generated using the Chromium Single Cell 3' Library \& Gel Bead Kit v.2 $(10 \times$ Genomics $)$ according to the manufacturer's protocol. Briefly, $1 \times 10^{5}$ viable cells were FACS-sorted using the LIVE/DEAD Aqua stain (Sigma), washed once in cold PBS, counted on the Countess II Automated cell counter (Thermo Fisher), and used to generate single-cell gel-beads in emulsion. The gel-beads in emulsion were disrupted after reverse transcription and the barcoded cDNA isolated and amplified by PCR. The resulting PCR products were fragmented, followed by end-repair, A-tailing and, adding sample indexes. The single-cell libraries were sequenced at a depth of 1 million reads per cell at the University of Edinburgh Genomics core facility on an Illumina NovaSeq machine.

Single-cell RNA-sequencing analysis. Alignment, filtering, barcode and unique molecular identifier (UMI) counting were performed using Cell Ranger v.2.1.0 (10 × Genomics) based on the human (GRCh38, Gen- 
code) and Toxoplasma (ToxoDB-39) genomes. The UMI count matrices from exposed and unexposed conditions were processed separately using the Seurat package v $2^{34}$. Briefly, monocytes expressing less than 200 genes and with $8 \%$ mitochondrial genes were excluded. Similarly, genes detected in $<10$ cells were excluded from further downstream analyses. Raw UMI counts were log-normalized and cells having a total normalized expression value three standard deviations from the mean were excluded. Additionally, cells outside the 95\% confidence interval of the total normalized UMI vs. number of genes per cell were filtered out. Based on the remaining samples, genes were scaled to remove the effects of library size and percent mitochondrial reads. Dimensionality reduction of the filtered genes was performed using the most significant principle components (PCs) based on Jackstraw permutations. The number of significant PCs varied with monocyte conditions, with 21 and 17 PCs in the unexposed and Toxoplasma-exposed monocytes, respectively. Clustering was performed using the Smart local moving (SLM) algorithm ${ }^{35}$. To generate the $t S N E$ plots of the combined exposed and unexposed monocytes data, the 18 significant PCs from the combined dataset were used, which were based on the normalized and scaled expression matrix of all cells filtered for each condition.

Toxoplasma cells were filtered based on a minimum expression of 30 genes, while genes expressed in less than 3 cells were removed. Subsequently this data was normalized, scaled and significant PCs calculated. The 5 most significant PCs were used in dimensionality reduction of the Toxoplasma gene expression data. Functional enrichment for differentially expressed genes were performed either using ToppFun from the ToppGene suite ${ }^{36}$ or ToxoDB ${ }^{31}$.

Cell sorting. $2 \times 10^{6}$ monocytes from three donors were separately seeded in 6-well cell culture plates and allowed to settle overnight. Freshly prepared parasites were then added to the cells at a MOI of 1 , briefly centrifuged, and incubated for an additional $1 \mathrm{~h}$ at $37^{\circ} \mathrm{C}$ with $5 \% \mathrm{CO}_{2}$. The cells were collected, washed once with PBS, resuspended in blocking buffer (PBS supplemented with $1 \%$ Goat serum) and incubated on ice for $30 \mathrm{~min}$. The cells were centrifuged at $400 \times \mathrm{g}$ for $4 \mathrm{~min}$ at $4{ }^{\circ} \mathrm{C}$ and washed once in ice-cold FACS buffer. CD98 $8^{+}$were sorted in BD FACSAria III (BD Biosciences) after double staining with Alexa Flour 488 anti-human CD98 (Novus Biologicals) monoclonal antibodies and Zombie Violet viability dye (Biolegend).

Cytokine and chemokine measurements. Sorted CD98 ${ }^{+}$and CD98 monocytes were seeded in fresh media and incubated for $4 \mathrm{~h}$ before collecting cell-free supernatants, which were stored at $-80{ }^{\circ} \mathrm{C}$ until use. Cytokines and chemokines, including IL1 $\beta$, IL12 and CCL2, were measured in supernatants using a multiplex Human Cytokine Array C3 kit (eBioscience) according to the manufacturer's protocol.

Statistical analysis. Differential expression of genes across groups of monocyte and Toxoplasma subsets was done using Wilcoxon test. Comparison of the number of genes or reads between groups was conducted using a one-way ANOVA, following a post-hoc test. To identify any enrichment in monocyte or Toxoplasma subsets, the Fischer's exact test was used. Where there was multiple testing, adjusted FDR was used.

Received: 6 January 2020; Accepted: 18 November 2020

Published online: 03 December 2020

\section{References}

1. Moser, R. J., Reverter, A. \& Lehnert, S. A. Gene expression profiling of porcine peripheral blood leukocytes after infection with Actinobacillus pleuropneumoniae. Vet. Immunol. Immunopathol. 121, 260-274 (2008).

2. Thi, E. P., Lambertz, U. \& Reiner, N. E. Sleeping with the enemy: how intracellular pathogens cope with a macrophage lifestyle. PLoS Pathog. 8, e1002551 (2012).

3. Avraham, R. et al. Pathogen cell-to-cell variability drives heterogeneity in host immune responses. Cell 162, 1309-1321 (2015).

4. Drayman, N., Patel, P., Vistain, L. \& Tay, S. HSV-1 single cell analysis reveals anti-viral and developmental programs activation in distinct sub-populations. Elife https://doi.org/10.7554/elife.46339 (2019).

5. Rato, S., Rausell, A., Muñoz, M., Telenti, A. \& Ciuffi, A. Single-cell analysis identifies cellular markers of the HIV permissive cell. PLoS Pathog. 13, e1006678 (2017).

6. Lin, P. L. et al. Sterilization of granulomas is common in active and latent tuberculosis despite within-host variability in bacterial killing. Nat. Med. 20, 75-79 (2014).

7. Lieberman, T. D. et al. Genomic diversity in autopsy samples reveals within-host dissemination of HIV-associated Mycobacterium tuberculosis. Nat. Med. 22, 1470-1474 (2016).

8. Balaban, N. Q., Gerdes, K., Lewis, K. \& McKinney, J. D. A problem of persistence: Still more questions than answers?. Nat. Rev. Microbiol. https://doi.org/10.1038/nrmicro3076 (2013).

9. Dubey, J. P. \& Beattie, C. P. Toxoplasmosis of Animals and Man (CRC Press, Boca Raton, 1988).

10. Tosh, K. W. et al. The IL-12 response of primary human dendritic cells and monocytes to toxoplasma gondii is stimulated by phagocytosis of live parasites rather than host cell invasion. J. Immunol. 196, 345-356 (2016).

11. Gazzinelli, R. T. et al. Parasite-induced IL-12 stimulates early IFN-gamma synthesis and resistance during acute infection with Toxoplasma gondii. J. Immunol. 153, 2533-2543 (1994).

12. Dupont, C. D. et al. Parasite fate and involvement of infected cells in the induction of CD4+ and CD8+ T cell responses to Toxoplasma gondii. PLoS Pathog. 10, e1004047 (2014).

13. Zhao, Y., Marple, A. H., Ferguson, D. J. P., Bzik, D. J. \& Yap, G. S. Avirulent strains of Toxoplasma gondii infect macrophages by active invasion from the phagosome. Proc. Natl. Acad. Sci. 111, 6437-6442 (2014).

14. Koshy, A. A. et al. Toxoplasma co-opts host cells it does not invade. PLoS Pathog. 8, e1002825 (2012).

15. Gov, L., Schneider, C. A., Lima, T. S., Pandori, W. \& Lodoen, M. B. NLRP3 and potassium efflux drive rapid IL-1 $\beta$ release from primary human monocytes during Toxoplasma gondii Infection. J. Immunol. 199, 2855-2864 (2017).

16. Christian, D. A. et al. Use of transgenic parasites and host reporters to dissect events that promote interleukin-12 production during toxoplasmosis. Infect. Immun. 82, 4056-4067 (2014). 
17. Safronova, A. et al. Alarmin S100A11 initiates a chemokine response to the human pathogen Toxoplasma gondii. Nat. Immunol. 20, 64-72 (2019).

18. Pandori, W. J. et al. Toxoplasma gondii activates a Syk-CARD9-NF-kB signaling axis and gasdermin dindependent release of IL-1 $\beta$ during infection of primary human monocytes. PLoS Pathog. 15, e1007923 (2019).

19. Saliba, A. E., Westermann, A. J., Gorski, S. A. \& Vogel, J. Single-cell RNA-seq: advances and future challenges. Nucleic Acids Res. 42, 8845-8860 (2014).

20. Kok, Y. L., Ciuffi, A. \& Metzner, K. J. Unravelling HIV-1 latency, one cell at a time. Trends Microbiol. 25, 932-941 (2017).

21. Reid, A. J. et al. Single-cell transcriptomics of malaria parasites. Doi.Org. 105015 (2017). https://doi.org/10.1101/105015.

22. Poran, A. et al. Single-cell RNA sequencing reveals a signature of sexual commitment in malaria parasites. Nature 551, 95-99 (2017).

23. Westermann, A. J., Gorski, S. A. \& Vogel, J. Dual RNA-seq of pathogen and host. Nat. Rev. Microbiol. 10, 618-630 (2012).

24. Steuerman, Y. et al. Dissection of influenza infection in vivo by single-cell RNA sequencing. Cell Syst. 6(679), e4-691.e4 (2018).

25. Avital, G. et al. scDual-Seq: mapping the gene regulatory program of Salmonella infection by host and pathogen single-cell RNAsequencing. Genome Biol. 18, (2017).

26. Satija, R., Farrell, J. A., Gennert, D., Schier, A. F. \& Regev, A. Spatial reconstruction of single-cell gene expression data. Nat. Biotechnol. 33, 495-502 (2015).

27. Villani, A.-C. et al. Single-cell RNA-seq reveals new types of human blood dendritic cells, monocytes, and progenitors. Science https://doi.org/10.1126/science.aah4573 (2017).

28. Grage-Griebenow, E., Flad, H. D. \& Ernst, M. Heterogeneity of human peripheral blood monocyte subsets. J. Leukoc. Biol. https ://doi.org/10.1189/jlb.69.1.11 (2001).

29. Gov, L., Karimzadeh, A., Ueno, N. \& Lodoen, M. B. Human innate immunity to Toxoplasma gondii is mediated by host caspase-1 and ASC and parasite GRA15. MBio 4, e00255 (2013).

30. Lee, A.-H., Iwakoshi, N. N. \& Glimcher, L. H. XBP-1 regulates a subset of endoplasmic reticulum resident chaperone genes in the unfolded protein response. Mol. Cell. Biol. https://doi.org/10.1128/mcb.23.21.7448-7459.2003 (2003).

31. Gajria, B. et al. ToxoDB: an integrated Toxoplasma gondii database resource. Nucleic Acids Res. 36, D553-D556 (2008).

32. Edfors, F. et al. Gene-specific correlation of RNA and protein levels in human cells and tissues. Mol. Syst. Biol. (2016). https://doi. org/10.15252/msb.20167144.

33. Perregaux, D. G., Laliberte, R. E. \& Gabel, C. A. Human monocyte interleukin-1 $\beta$ posttranslational processing. Evidence of a volume-regulated response. J. Biol. Chem. (1996). https://doi.org/10.1074/jbc.271.47.29830.

34. Butler, A., Hoffman, P., Smibert, P., Papalexi, E. \& Satija, R. Integrating single-cell transcriptomic data across different conditions, technologies, and species. Nat. Biotechnol. https://doi.org/10.1038/nbt.4096 (2018).

35. Waltman, L. \& Van Eck, N. J. A smart local moving algorithm for large-scale modularity-based community detection. Eur. Phys. J. B https://doi.org/10.1140/epjb/e2013-40829-0 (2013).

36. Chen, J., Bardes, E. E., Aronow, B. J. \& Jegga, A. G. ToppGene Suite for gene list enrichment analysis and candidate gene prioritization. Nucleic Acids Res. https://doi.org/10.1093/nar/gkp427 (2009).

\title{
Acknowledgements
}

The authors thank the Baillie and Fitzgerald labs at the Roslin Institute for help with the blood sampling approvals and protocols. We also wish to thank the University of Edinburgh Genomics Core for providing facilities and services. J.A was supported by Wellcome Trust grant 201531/Z/16/Z. M.A.H is funded by a University of Edinburgh Chancellor's Fellowship and a Bill and Melinda Gates Foundation award to the Centre for Tropical Livestock Genetics and Health (OPP1127286). The Roslin Institute receives strategic investment funding from the Biotechnology and Biological Sciences Research Council.

\section{Author contributions}

M.A.H, P.R and N.C.H conceived and designed the experiments. M.A.H, P.R, A.P, and A.G performed the experiments and analysed the data. J.A, T.C.F, and M.W contributed materials and analysis tools. A.P, T.C.F, and M.A.H wrote the paper.

\section{Competing interests}

The authors declare no competing interests.

\section{Additional information}

Supplementary information is available for this paper at https://doi.org/10.1038/s41598-020-78250-0.

Correspondence and requests for materials should be addressed to M.A.H.

Reprints and permissions information is available at www.nature.com/reprints.

Publisher's note Springer Nature remains neutral with regard to jurisdictional claims in published maps and institutional affiliations.

\begin{abstract}
Open Access This article is licensed under a Creative Commons Attribution 4.0 International License, which permits use, sharing, adaptation, distribution and reproduction in any medium or
format, as long as you give appropriate credit to the original author(s) and the source, provide a link to the Creative Commons licence, and indicate if changes were made. The images or other third party material in this article are included in the article's Creative Commons licence, unless indicated otherwise in a credit line to the material. If material is not included in the article's Creative Commons licence and your intended use is not permitted by statutory regulation or exceeds the permitted use, you will need to obtain permission directly from the copyright holder. To view a copy of this licence, visit http://creativecommons.org/licenses/by/4.0/.
\end{abstract}

(c) The Author(s) 2020 